\title{
Literary Language in Baoding Old Troupe
}

\author{
Zijuan $\mathrm{Li}^{1}$, Shan Wang ${ }^{1}$ \\ ${ }^{1}$ XingTai University, Xingtai, 054001, China
}

Keywords: Baoding old troupe; Libretto; Literary language

\begin{abstract}
Baoding old troupe is the most representative opera art in Baoding area, and its libretto shows unique style of literary language with the change in vocal music and territorial culture. Its language originates from local board drum; after more than 100 years of changes and development as well as several generations of old troupe artists' unremitting efforts, Baoding old troupe forms concise, lucid, simple, and healthy feature with the temperament of Yan and Zhao State.
\end{abstract}

\section{Introduction}

With 3,000-year brilliant culture and history, the ancient city Baoding is the old place of Yan and Zhao State in ancient China, and the word "Yan State in South and Zhao State in North" is a historic summary for geographic position of Baoding. Those tangible cultural relics and historic sites contain rich cultural connotation, and they tell historic fluctuation and rising and declining of dynasties. All of those provide rich historic materials and singing materials for opera culture in Baoding. Baoding old troupe is the most representative opera art in Baoding area, and the connotation of literary language owned by its libretto reflects the rich color of territorial culture in Baoding area.

\section{Origin of libretto}

Most of contents of Baoding old troupe in early stage are describing the vivid stories about greenwood heroes and national heroes. Therefore, the language of old troupe shows magnificent color of Yan and Zhao culture. The character depiction with great momentum is shown via troupe language to vividly show the most outstanding chant in a heroic but mournful tone and national temperament on the troupe stage. In Kong Li's He Hua Dian, it can be seen that it has consistency with Baoding old troupe according to the language feature, and most of the language are the deposit of Baiyangdian culture. However, Baiyangdian (Xidian) is the initial origin of Baoding old troupe.

What the old Baoding people most like to hear is Baoding old troupe, and this feeling becomes stronger and stronger as the time goes by. The reason may be the libretto. The literary connotation of libretto of Baoding old troupe is not only fresh and plain, but also heavy and bold. It is mellow wine and can withstand the sedimentation in the time. Actually speaking, we like the "tune" sung in Baoding people's mouth - Baoding old troupe, and it is the story-telling artists' libretto that makes me know it. One of important sources of libretto of Baoding old troupe is the libretto of drum story, and mainly the libretto of local board drum.

The initial understanding on the features of libretto of Baoding old troupe originates from the board drum in local village fair; the board drum is a kind of folk art form popular in Hebei area, especially in village area of Hebei. The feature is that the performer generally takes the board on the left hand and takes drum stick on the right hand for talking and singing with a standing posture; in this process, the performer combines with hitting board and drum in succession for convenience of combination of talking and singing, and another performer specially make accompaniment with Sanxian (a three-stringed plucked instrument). The board drum is the main way of entertainment for peasants in slack season in the early agrarian age; it includes talking and singing with rough and plain style. The initial content mainly involves historical stories and tells hero figures; the board drum is formed in the end of Ming Dynasty and slightly earlier than Baoding old troupe. The representative works include the Investiture of the Gods, Generals of the Yang Family in Northern Song Dynasty, and Three Heroes and Five Gallants, etc. The local artists and singers tell the stories they hear from 
story-telling artists in the fair and match them with local melody by virtue of their intelligent comprehension and creativity to compose them into libretto to make the libretto become bright, clear, and plain and jump with the melody, thus it can move people deeply. The rudiment of libretto is arbitrarily dragged from drum artists. To some sense, they are brilliant artists; they combine drum stories with Baoding old troupe to make the language of Baoding old troupe have certain feature of literary story and become closer to life, and Baoding old troupe also uses many songs from drum stories. Those librettos become the most beautiful sound memory of some old people, and they reveal a kind of plain warmness.

\section{Magnificence and gentleness}

Baoding old troupe is one of the most representative and influential local troupes. It was the folk song on village flower fair in Baiyangdian area - Hexi tune in the beginning, and then basically formed its features in Daoguang and Xianfeng's period in Qing Dynasty. In the central Hebei, people will croon several libretto they most like, and Baoding old troupe is almost close to the dialect; it is plain and natural, with the fragrance of soil. Therefore, its libretto has the popularity of folk literary. Baoding old troupe can be also called old troupe, and it belongs to a branch of Hebei opera; it inherits the resounding and cool of Hebei opera and it is mainly popular in Shijiazhuang, Cangzhou, Beijing, and Zhangjiakou of Hebei province, with bright and strong local color and village smell, all of which endows the libretto with heavy historical literariness.

As an artistic miracle with unique feature in Hebei local operas, Baoding old troupe, as traditional local art, attracts more and more attention in recent years. Through reference and absorption to different historical periods, its libretto forms visual, vivid, lucid, and concise language feature and applies lots of metaphor techniques. For example, after Pan Renmei was overpowered by the young person Hu Pixian, there is a sentence: "Pan Renmei is detained in the border, and a small rat eats a fat wolf.” This sentence applies vivid metaphor to fully show Pan Renmei's insolent, rude, imperious, and domineering image. This shows that Baoding old troupe forms its healthy, plain, and bold language style on the basis of inheriting traditional operas. Meanwhile, according to the content requirement of traditional operas, its libretto is of slightly strong and loyal, generous language color of north China. The libretto of Baoding old troupe shows its moving literary charm according to the change in opera melody, and this literary charm originates from the situation that it has deep mass base, and also has fresh and vigorous artistic vitality.

An audience once said that there was a saying in the folk that "the cooking can't be separated from pot and stove, and listening to the opera can't be separated from old troupe"; one half was vocal music, and one half was libretto. The main reason why Baoding old troupe occupies a high position in rural people's heart in central Hebei is its excellent libretto.

As for the old troupe in early period, due to simple and crude condition in stage equipments, costumes, and props, in order to win the audience's praise, except for the performer's singing level, the libretto plays a key role. Therefore, at the beginning, the old troupe pays great attention to the materials of libretto, and the historical allusions and classical literature in the libretto are more prominent compared to other operas, for Baoding old troupe mainly draws materials from historical stories in development period. The singing tune is divided into male tune and female tune. In terms of libretto, the male tune is passionate and the female tune is gentle; when people mention the old troupe, they will think of the symbolic male tune. Because the sound range of male sound is incapable of high-pitch singing in old troupe, the male tune has to be sung by female performers; the famous old troupe player Wang Guanying is just a female player. In the old troupe, people pay more attention to male tune than female tune, thus the representative tune is always male tune. Therefore, the libretto feature of Baoding old troupe is mainly bold, unconstrained, and magnificent. Due to the fact that the tune simulates the pronunciation effect of local dialect, the melody trend of tune is very similar to local dialect, especially similar to the dialect around the place of formation of old troupe Baiyangdian, thus the singing language is more plain and nature compared to other operas. It can be said that most of librettos of old troupe utilize the voice structure of local dialect to be sung out in an exaggerated 
way. Therefore, when the local audiences listen to the old troupe, they feel very cordial and can understand the content sung by performers, thus the old troupe is widely popular. The libretto of Baoding old troupe inherits excellent Chinese traditional culture; compared to other operas in north China, Baoding old troupe retains certain similarity. According to the operas on performance in 1959, there are more than 200 names of tunes, most of which inherits the names of tunes of Hebei opera, Beijing opera, and Kunqu opera. The common names of tunes of style of funeral music include Suo $\mathrm{Na} \mathrm{Pi}$, Meng Jing, Da Ji Sheng Cao, and Ku Huang Tian; the names of tunes of style of joyful music includes Hai Qing Ge, Dong Fang Zan, Da Man Tang Hong, and Xiao Man Tang Hong; the names of tunes of style of military music include Jiang Er Shui, Kai Men Ge, and Shui Long Yin; the names of tunes of style of banquet music include Di Liu Zi, Da Kai Men, San Bu Ge, and Bai Yan Qu; besides, there are also Wa Wa, Bei Ma Ling, Luo Gu Jing, and other names. Those names of tunes are closely related to ancient poems, thus the libretto of Baoding old troupe combines with the rhythm features of ancient Chinese poetry. Take Li Si and Zhao Gao: Persuade the Madam to Bear the Pain and Not Cry sung by the old troupe performing artist Wang Guanying as an example: the libretto is shown as below:

"I persuade you, my wife, to bear the pain and not cry; the ups and downs in the official circle are common things.

I have had no regard for my life and death at this time, and I am only concerned with my family members.

I am afraid that it is hard to escape from Zhao Gao's hands, and I only hope that you can be more cautious and take precautions against any danger."

It is obvious that the rhyme is applied at the end of sentence; in this way, the libretto is fluent, natural, and powerful.

Except for magnificence, the libretto of Baoding old troupe also has an aspect of gentleness. There is a very famous lake called "bright pearl in north China" in the central Hebei plain; this lake is Baiyangdian lake, and the local people also call it as "Xidian”. About in Yuan and Ming Dynasty, a kind of opera called "Hexi Tune" became popular from an unknown person. The elegant, beautiful, and euphemistical libretto was plain and full of charm, just like lotus in the lake and also like industrious, kind-hearted, and beautiful fishing girls in the lake. Then, Those librettos and tunes gradually became an important voice in the major affairs of the peasant families and fishermen's families, such as flower fair in the lunar January, birth of a boy in a rich family, old person's birthday party, and weddings and funerals, so that "the things can't be done without tunes". At that time, there was no more spiritual life in agrarian age, and the libretto produced with "Hexi Tune" became an abundant spirit banquet for peasants and fishermen along Baiyangdian Lake.

At this time, Han Dacang, a crucial person who developed "Hexi Tune" into opera, appeared in Hexi tune. Later, Hexi tune was not merely some tunes, but evolved into a series of vocal music. The feature of its libretto also changed accordingly; the libretto was not limited to the peasant families' weddings and funerals in Baiyangdian any more, but faced to the wide space of society and history, and the libretto changed from gentle style to magnificent style. The change of libretto promoted the development of the opera named "old troupe" which gradually appeared in people's vision.

After inheriting Han Dacang's achievements, Zhou Fucai pushed the old troupe to a new high peak by virtue of the opera Diao Kou and Quan Jun; finally, the professional class of old troupe was birth in the period of Emperor Guangxu in Qing Dynasty; then, the old troupe quickly entered into Baoding and other cities and became popular in urban people. In the 1930s, the creation of libretto of old troupe entered into a prosperous time. In the cities, there were various kinds of art; in particular, Baoding was close to Beijing, which was a holy land under the emperor's foot; the artists who wanted to enter into Beijing all over the country stayed here. With increase of chances of art exchange and learning, the development of libretto of old troupe was very quick, and the libretto changed from straightforward style to diversified style, and the description on scenery and psychology was more exquisite, which made description of artistic conception, expression of thoughts and feelings, and shaping of figure image become more bright and vivid. According to the libretto of old troupe 
performing artist Han Wenmei’s Red-cloth Fairy - Follow My Father's Order, we can see the change in libretto:

"I follow my father's order to go to the capital in order to offer birthday felicitations to my uncle.

When I leave from home, I feel very happy.

It is just like the situation that a bird goes out of the cage.

On the road, I can appreciate endless beautiful scenery.

It seems that the sky is linked with the water and the water is also linked with the sky, with mountains around.

Paris of wild ducks float on the water.

They are sometimes sunk into the water and sometimes fly in the sky.

The water is so clear that I can see swimming fishes in the water.

The grass is interweaved together.

When I raise my eyes, I find that a rainbow-shaped bridge appears before my eyes.

While I continue my journey, I hear the sound of gongs and drums from somewhere."

The "home" of old troupe is in Baiyangdian of Hebei, a place where Hebei opera is very popular, thus the libretto also has the resounding and agitation of opera music to show the roughness and heroic feature, and also shows the beauty of water villages and the winding charm. The old troupe both has north Chinese people's strong and straightforward personality, and also has the exquisite and beautiful image, thus it lets people enjoy themselves so much as to forget to leave.

\section{Temperament of Yan and Zhao State}

The basic structure of libretto of Baoding old troupe belongs to the above and the following sentence of talking and singing genre; deeply influenced by local drum words, the libretto has strong narrativity and the feature of north China talking and singing literary which can stand for Central Plain Culture. Through comparison between the libretto of Shaoxing opera in South China and the libretto of Baoding old troupe, it can be found the libretto of Shaoxing opera is more like beautiful verse, that is, ancient poetry. This is because Shaoxing opera is produced in Wu State, and it draws the materials from folk life and it is more beautiful and exquisite. The pronunciation of its libretto is similar to the pronunciation of dialect of Shengxian County (place of birth of Shaoxing opera), which makes the libretto become more charming. We can appreciate its style from Liang Shanbo and Zhu Yingtai:

"There is a younger sister in my family, and she is clever and quick-witted.

She is good at embroidery, calligraphy and painting."

Baoding old troupe is produced in the old place of Yan and Zhao state where there are often sad melody since ancient time, and its content mainly draws materials from the historical stories of princes and marquises, generals and ministers of state, and greenwood heroes; the pronunciation of libretto combines with the tune of dialect in central Hebei and the libretto shows more ungrudging narration with the trend of tune. In the libretto, most of sentences are 10-character and 7-character sentence, and the antithesis, parallelism, allusion, and other forms are applied to show heroic and bold temperament. Through the libretto of classic old troupe Loyal To Imperial Family, we can feel this style, and the libretto is shown as below:

"A virtuous person can control the world and be praised by all people, and a person without virtues will lose its court and let the masses have no means to live.

You only know to be biased by flattery, but you don't see their wild ambition under their smiles.

You close your ears to sincere advices, but blame that your officials are not sincere.

You cline to wine and women, waste affairs of state, keep close to evils, and leave far away from virtuous court officials.

You behead excellent general and hound your teacher Wang Yanling to death.”

However, with the time development, more changes have happened in some modern operas which are not limited to one style; however, they always can't be separated from the plain narrativity of talking and singing literary. 


\section{Conclusion}

The literary language of Baoding old troupe stands for some features of Yan and Zhao culture, desolate, plain, heroic, bold, and euphemistic; Baoding old troupe is just a perfect combination of literary and local opera art and it tells history and culture. What makes us feel relieved is that Baoding old troupe was listed into the $1^{\text {st }}$ batch of list of intangible cultural heritage protection in Hebei province in 2006, and the libretto created in this period shows deeper ideological contents, bright sense of the time, and colorful breath of life. We hope that this flower of opera literary can be rooted in the culture soil of central Hebei and can be in full bloom!

\section{Acknowledgments}

This paper is the project of Hebei social science fund project Survey on Development Status of Baoding Old Troupe and Research on Protection Countermeasures, and the project number is HB13YS008.

\section{References}

[1] Li Zhongqi: Brief History of Old Troupe, Chinese Drama Publishing House, 1985.

[2] Collection of Chinese Opera Music - Old Troupe Sub-volume of Hebei Volume, collection of Baoding opera music editing group. 